\title{
Countervailing Measures und das Chinesische Beitrittsprotokoll zur WTO
}

\author{
Marc Bungenberg und Pieter Van Vaerenbergh"
}

Inhalt

A. Einleitung: Notwendigkeit für stärkere „Werkzeuge“ 268

B. Ausgangspunkt für eine neue alternative Methodik: Das chinesische $\begin{array}{ll}\text { Beitrittsprotokoll zur WTO } & 270\end{array}$

C. Antisubventionsuntersuchungen der EU gegen China 273

I. Allgemeiner Überblick über die handelspolitischen Schutzinstrumente der EU

II. Handelsschutzinstrumente und staatlich verzerrte Märkte 274

III. Aktueller Einsatz von Antisubventionsuntersuchungen gegen China 275

IV. Allgemeine Durchführung von EU-AS-Untersuchungen 277

D. Praktische Schwierigkeiten bei China betreffenden

Antisubventionsuntersuchungen

E. Erste Ideen für neue Antisubventionsuntersuchungsmethoden bei

Chinesischen Importen

I. Anwendung von Abschnitt 15(b) CAP, kombiniert mit einer Verlagerung der Beweislast

1. Die Bedingung: „Besondere Schwierigkeiten“ 282

2. Anpassungen der Bemessungsgrundlagen 284

3. Die alternative Bemessungsgrundlage: Preise außerhalb des Landes 284

II. Weitere nicht vom CAP abgedeckte Maßnahmen zur Effektivierung des Antisubventionsmechanismus

F. Schlussfolgerungen und Ausblick 288

$\begin{array}{ll}\text { Annexe } & 291\end{array}$

* Prof. Dr. Marc Bungenberg LL.M. (Lausanne) ist Direktor des Europainstituts und Inhaber des Lehrstuhls für Offentliches Recht, Völkerrecht und Europarecht an der Universität des Saarlandes. Pieter Van Vaerenbergh LL.M. ist wissenschaftlicher Mitarbeiter und Doktorand am Lehrstuhl von Prof. Dr. Marc Bungenberg LL.M. (Lausanne) an der Universität des Saarlandes. Der vorliegende Beitrag beruht auf einem wissenschaftlichen Gutachten, das Marc Bungenberg zusammen mit Pieter Van Vaerenbergh in Auftrag von der Grünen/EFA-Fraktion im Europäischen Parlament erstattet hat. Der Inhalt dieses Papiers repräsentiert die persönlichen Ansichten der Autoren und nicht die der Grünen/EFA-Fraktion und sollte nicht als Repräsentation irgendeiner Form politischer Unterstützung ausgelegt werden. 


\section{A. Einleitung: Notwendigkeit für stärkere „Werkzeuge“}

Die bestehenden Instrumente der Europäischen Union (EU) bieten nur unzureichend die Möglichkeit, auf die Auswirkungen der von Drittstaaten gewährten Subventionen auf den EU-Binnenmarkt zu reagieren. Allerdings wird es als notwendig erachtet, sich gerade gegen solche Subventionen der Industrie in Drittländern zur Wehr zu setzen, die sich „innerunional“ gravierend bemerkbar machen.

Die bisher in der EU in Kraft getretenen Maßnahmen einer neuen Industriepolitik umfassen ein Screening-Verfahren für ausländische Investitionen ${ }^{1}$ und Leitlinien für die Beteiligung von Bietern aus Drittländern an öffentlichen Ausschreibung. ${ }^{2}$ Für die Durchsetzung einer neuen Industriepolitik können weiter u.a. die extraterritoriale Anwendung der EU-Beihilferegeln, die Schaffung von „European Champions“, die Verabschiedung eines International Procurement Instruments ${ }^{3}$ und die Verweigerung der Genehmigung von mit staatlicher Unterstützung finanzierter Fusionen in Betracht gezogen werden. ${ }^{4}$ Darüber hinaus wird - zuletzt in der neuen unionalen Industrie-

1 VO (EU) 452/2019, zur Schaffung eines Rahmens für die Überprüfung ausländischer Direktinvestitionen in der Union, ABl. L 79 v. 21.03.2019, S. 1 ff; vgl. hier zuletzt auch die Mitteilung der Kommission vom 25. März 2020, Leitlinien für die Mitgliedstaaten betreffend ausländische Direktinvestitionen, freien Kapitalverkehr aus Drittländern und Schutz der strategischen Vermögenswerte Europas im Vorfeld der Anwendung der Verordnung (EU) 2019/452 über die Überprüfung ausländischer Direktinvestitionen, C(2020) 1981, abrufbar unter: https://data.consilium.europa.eu/doc/document/ST-7028-2020-INIT/de/pdf (deutsch, ohne Anlage) bzw. https://trade.ec.europa.eu/doclib/docs/2020/march/tradoc_15 8676.pdf (11.03.2020).

2 Europäische Kommission, Leitlinien zur Teilnahme von Bietern und Waren aus Drittländern am EU-Beschaffungsmarkt, C(2019) 5459 final, 24.07.2019.

3 S. Europäische Kommission, Instrument betreffend das öffentliche Beschaffungswesen, S. 1 (abrufbar unter: https://op.europa.eu/de/publication-detail/-/publication/4545bd05-574c11e9-a8ed-01aa75ed71a1/language-de/format-PDF (11.03.2020); Europäische Kommission, Eine funktionierende öffentliche Auftragsvergabe in und für Europa, COM(2017) 572 final v. 03.10.2017, S.12; Europäische Kommission, EU-China - Strategische Perspektiven, JOIN(2019)5 final v. 12.03.2019, S. 8; Europäische Kommission, Geänderter Vorschlag für eine Verordnung des Europäischen Parlaments und des Rates über den Zugang von Waren und Dienstleistungen aus Drittländern zum EU-Beschaffungsmarkt für öffentliche Aufträge und über die Verfahren zur Unterstützung von Verhandlungen über den Zugang von Waren und Dienstleistungen aus der Union zu den Märkten für öffentliche Aufträge von Drittländern, $\operatorname{COM(2016)~} 34$ final v. 29.01.2016; Europäische Kommission, Vorschlag für eine Verordnung des Parlaments und des Rates über den Zugang von Waren und Dienstleistungen aus Drittländern zum EU-Binnenmarkt für das öffentliche Beschaffungswesen und über die Verfahren zur Unterstützung von Verhandlungen über den Zugang von Waren und Dienstleistungen aus der Union zu den öffentlichen Beschaffungsmärkten von Drittländern, $\operatorname{COM}(2012) 124$ final v. 21.03.2012.

4 Europäische Kommission, EU-China-Strategische Perspektiven, Gemeinsame Mitteilung an das Europäische Parlament, den Europäischen Rat und den Rat, 12.03.2019, JOIN(2019) 5 final. 
strategie ${ }^{5}$ - die Notwendigkeit der Verabschiedung eines gestärkten Antisubventionsinstruments (AS) betont, das den eigentlichen Kern des Problems angeht: Subventionen, die von staatlichen chinesischen Unternehmen (Chinese State-Owned Enterprises, SOEs) kommen.

Das derzeitige Regelwerk für Antisubventionsmaßnahmen funktioniert zwar weitgehend, wenn die Einfuhren aus Marktwirtschaften kommen, ${ }^{6}$ aber es können nicht alle Konstellationen und alle Folgen mit insbesondere starken Einflüssen auf die Wirtschaft erfasst werden. Die Anwendung der bestehenden Regeln auf Subventionen an wie auch durch SOEs führt zu gravierenden Problemen.

Die EU - Kommission, Parlament und die Mitgliedstaaten - beabsichtigt daher insbesondere zu untersuchen, wie sie mit den verzerrenden Auswirkungen von ausländischem Staatseigentum und staatlicher Finanzierung ausländischer Unternehmen auf dem EU-Binnenmarkt angemessen umgehen könnte. ${ }^{7}$ Darüber hinaus drängt der USA-EU-Japan Trilog auf dieses Thema, ${ }^{8}$ ebenso wie andere Einrichtungen und Organisationen wie beispielsweise der Bundesverband der Deutschen Industrie (BDI). ${ }^{9}$

Eine wirksame und ausgewogene Anwendung der EU-Handelsschutzinstrumente (Trade Defence Instruments, TDI) mit einem verstärkten Antisubventionsinstrument ist dabei eine notwendige und wichtige Maßnahme zur Gewährleistung gleicher Wettbewerbsregeln. Es ist zu untersuchen, ob für die Zukunft wirksamere Handelsschutzinstrumente innerhalb der durch das Recht der Welthandelsorganisation (WTO) gesetzten Grenzen geschaffen werden können. Dieser Beitrag befasst sich im Folgenden allerdings ausschließlich mit dem Bereich der Antisubventionsmaßnahmen, insbesondere mit der Möglichkeit von Abschnitt 15(b) des chinesischen Beitrittsprotokolls zur WTO (China Accession Protocol, CAP), und erlaubt, das Instrument der Antisubventionsuntersuchungen und Ausgleichszölle in Bezug auf die Einfuhren aus China zu stärken. Bislang hatte es fast den Anschein, dass die Verpflichtungen Chinas aus dem Beitrittsprotokoll und die Möglichkeiten aller anderen Mitglieder der Welthandelsorganisation, auf bestimmte chinesische Maßnahmen zu reagieren, bewusst übersehen worden sind. Es wird vorgeschlagen, dass die EU Chinas Beitrittsverpflichtungen überprüfen sollte, um neue und durchsetzbare WTO-konforme Regeln

5 S. aktuell Europäische Kommission, Mitteilung der Kommission an das Europäische Parlament, den Europäischen Rat, den Rat, den Europäischen Wirtschafts- und Sozialauschuss und den Ausschuss der Regionen, COM(2020) 102 final, 10.03.2020; s. auch Mission Letter von President-elect of the European Commission Ursula von der Leyen to Commissionerdesignate for Trade Phil Hogan, 10.09.2019.

6 China wird nicht als Marktwirtschaft betrachtet. S. Resolution des Europäischen Parlaments vom 12. Mai 2016 zum Marktwirtschaftsstatus Chinas (2016/2667(RSP)), P8_TA(2016)02232, abrufbar unter: http://www.europarl.europa.eu/doceo/document/TA8-2016-0223_EN.pdf (11.03.2020).

7 Europäische Kommission, EU-China - Strategische Perspektiven, Gemeinsame Mitteilung an das Europäische Parlament, den Europäischen Rat und den Rat, 12.03.2019, JOIN(2019) 5 final, Maßnahme 8.

8 S. aktuell Joint Statement of the Trilateral Meeting of the Trade Ministers of Japan, the United States and the European Union, Washington, D.C., 14.01.2020, abrufbar unter: https://trad e.ec.europa.eu/doclib/docs/2020/january/tradoc_158567.pdf (11.03.2020).

9 Bundesverband der Deutschen Industrie, BDI Policy Papers China, Januar 2019. 
einzuführen, die insbesondere Wettbewerbsverzerrungen durch staatliche Unternehmen ins Visier nehmen. ${ }^{10}$ Insbesondere muss Abschnitt 15(b) CAP, der eine alternative Berechnungsmethode für Ausgleichszölle zulässt, neue Aufmerksamkeit geschenkt werden.

In diesem Papier wird zunächst das chinesische Beitrittsprotokoll als Teil des geltenden Rechtsrahmens für Antisubventionsuntersuchungen gegen Importe aus China hervorgehoben (B), sodann wird auf den derzeitigen Einsatz dieser Untersuchungen durch die EU gegen China eingegangen (C). Anschließend werden praktische Schwierigkeiten bei der Durchführung von Antisubventionsuntersuchungen gegen chinesische Importe, auf die die Kommission als Untersuchungsbehörde in der EU stößt, skizziert (D). Im thematischen Zentrum dieses Beitrages stehen die dann diskutierten Möglichkeiten für neue und alternative Ansätze für Gegenmaßnahmen gegen unzulässige Subventionen von chinesischen Produkten, die in die EU importiert werden, bevor die Voraussetzungen und erste Ideen für ein verstärktes AS-Instrument dargelegt werden (E). Dieses Papier schließt mit einer Schlussfolgerung (F).

\section{B. Ausgangspunkt für eine neue alternative Methodik: Das chinesische Beitrittsprotokoll zur WTO}

China gehörte zu den Unterzeichnern des GATT 1947 (General Agreement on Tariffs and Trade), zog sich aber bereits 1950 aus diesem zurück. Im Jahr 1986 beantragte China die GATT-Wiederaufnahme. Die Verhandlungen zwischen China und den GATT/WTO-Vertragsparteien dauerten 15 Jahre, bevor China im Jahr 2001 schließlich als WTO-Mitglied aufgenommen wurde.

Zum Zeitpunkt seines Beitritts wurde China als Staat in einer „Übergangsphase zur Marktwirtschaft" angesehen, aber noch nicht als wirkliche Marktwirtschaft. Im November 2001 hat China das Beitrittsprotokoll (Chinese Accession Protocol) ${ }^{11}$ akzeptiert und ist seit dem 11. Dezember 2001 Mitglied der WTO. Der Beitritt Chinas basiert daher insbesondere auf dem CAP. Dieses enthält Bestimmungen, die bereits Gegenstand langwieriger WTO-Streitverfahren waren. ${ }^{12}$

China hat sich im CAP zu besonderen Regeln verpflichtet, auf die die WTO-Mitglieder auch bei der Anwendung der Maßnahmen des Übereinkommens über Subventionen und Ausgleichsmaßnahmen gegen chinesische Importe zurückgreifen können. Diese Regeln betreffen die Ermittlung und Bemessung chinesischer Subventionen und bestimmen auch die Anfechtbarkeit von Subventionen die staatlichen Unterneh-

10 So auch Chad P. Bown, Anhörung vor der U.S.-China Economic and Security Review Commission, 08.06.2018.

11 Protocol on the Accession of the People's Republic of China, WT/L/432, 10. November 2001 und Report of the Working Party on the Accession of China, WT/CC/CHN/49, 01.10.2001, paras 150-151. Vietnam akzeptierte die gleichen Bedingungen beim Beitritt. S. Report of the Working Party on the Accession of Viet Nam, 27.10.2006, WT/ACC/VNM/ 48, para. 255.

12 Insb. der EC-Fasteners Entscheidung. S. Appellate Body report, European Communities - Definitive Anti-Dumping Measures on Certain Iron or Steel Fasteners from China, WT/ $\mathrm{DS} 397 / \mathrm{AB} / \mathrm{R}, 15.07 .2011$. 
men in China gewährt werden. Das CAP schafft hier vereinfachte Möglichkeiten für die Einführung von Ausgleichszöllen (Countervailing Duties, CDs) gegen Importe von chinesischen Staatsunternehmen. Daher muss verstärkt geprüft werden, ob das Antisubventionssystem ein zukünftig noch effizienteres Mittel gegen subventionsbedingte Marktverzerrungen im Zusammenhang mit chinesischen Importen werden kann, ${ }^{13}$ auch wenn teilweise argumentiert wird, dass Antisubventionsmaßnahmen schlicht ungeeignet sind, um den chinesischen Bedrohungen entgegenzuwirken. ${ }^{14}$ In diesem Zusammenhang ist jedoch festzustellen, dass Abschnitt 15(b) CAP noch nicht explizit angewendet und auch kaum diskutiert worden ist, obwohl diese Bestimmung neue Wege im Bereich der handelspolitischen Schutzinstrumente eröffnen könnte.

Abschnitt 15(b) CAP erlaubt insbesondere die Anwendung alternativer Methoden zur Identifizierung und Berechnung von Ausgleichszöllen.

\section{Price Comparability in Determining Subsidies and Dumping ${ }^{15}$}

Article VI of the GATT 1994, the Agreement on Implementation of Article VI of the General Agreement on Tariffs and Trade 1994 (“Anti-Dumping Agreement”) and the SCM Agreement shall apply in proceedings involving imports of Chinese origin into a WTO Member consistent with the following:

a) $[\ldots]$

b) In proceedings under Parts II, III and V of the SCM Agreement, when addressing subsidies described in Articles 14(a), 14(b), 14(c) and 14(d), relevant provisions of the SCM Agreement shall apply; however, if there are special difficulties in that application, the importing WTO Member may then use methodologies for identifying and measuring the subsidy benefit which take into account the possibility that prevailing terms and conditions in China may not always be available as appropriate benchmarks. In applying such methodologies, where practicable, the importing WTO Member should adjust such prevailing terms and conditions before considering the use of terms and conditions prevailing outside China.

c) $[\ldots]$

d) $[\ldots]$

13 Müller, Taming the Dragon, S. 4.

14 Aegis Europe, A Pragmatic Approach To China MES: Wait for the WTO to Decide. Why "mitigation options" don't work, the risks of a unilateral interpretation of the Protocol and the key pillars of an effective anti-dumping system, abrufbar unter: https://static1.squares pace.com/static/5537b2fbe4b0e49a1e30c01c/t/56950ffca12f44b2eb0fc7b2/1452609533236 /A+PRAGMATIC+APPROACH+TO+CHINA+MES.pdf (11.03.2020): "For a number of reasons, it is unrealistic to expect that the anti-subsidy instrument would 'fill the gap' left by the ineffectiveness of anti-dumping measures following a grant of MES to China.

To begin with, the subsidies agreement was drafted to deal with conditions in a market economy and is ill-suited to deal with the economy-wide and fundamental distortions inberent in a planned economy like China's. Prices for key industrial inputs and end products in China's economy are shaped by government policy, are very low, and take no account of market conditions. An artificially low price, as such, is not a subsidy whereas under the $A D$ Agreement, it is an unfair market distortion which is addressed by making reference to the full costs of production."

15 Abschnitt 15 CAP. 
Abschnitt 15(b) CAP scheint in etwa dem Muster der Ad Note zu Artikel VI:1 GATT zu folgen, in der anerkannt wird, dass das einführende Mitglied es unter bestimmten Umständen für notwendig erachten könnte, "to take into account the possibility that a strict comparison with domestic prices in such a country may not always be appropriate." Als solches erweitert Abschnitt 15(b) CAP die Logik der Ad Note auf die WTO-Antisubventionsmaßnahmen. Es ist die einzige WTO-Bestimmung, die die Verwendung alternativer Vergleichsgrößen bei der Berechnung der Höhe einer vom chinesischen Staat gewährten Subvention ausdrücklich erlaubt.

Im Gegensatz zu den Abschnitten über Antidumpingmaßnahmen sind keine Fristen für die Anwendbarkeit dieser Vorschrift vorgesehen. Zwar wird teilweise argumentiert, dass es sich hierbei nur um einen redaktionellen Fehler handeln könne und dass auch Abschnitt 15(b) CAP auslaufen müsse; ${ }^{16}$ eine solche Auslegung würde jedoch dem insoweit eindeutigen Wortlaut der Vorschrift widersprechen. Die Verfasser hatten gerade nicht die Absicht, diesen Abschnitt auslaufen zu lassen. Die Verhandlungsgeschichte zeigt, dass es sich nicht um einen redaktionellen Fehler, sondern um eine bewusste Formulierung handelt. ${ }^{17}$ Daher können sich Untersuchungsbehörden wie die Kommission jederzeit auf diese Vorschrift stützen.

Diese Bestimmung gilt für alle chinesischen Subventionen. In der Praxis erlaubt diese Bestimmung unabhängig von der Art der Subvention und der subventionierenden Behörde, die Inlandspreise bei der Berechnung der jeweiligen Begünstigung außer Acht zu lassen und Anpassungen vorzunehmen oder andere ausländische Preise zu verwenden. Dies kann der Kommission eine umfassende Grundlage für die Handhabung chinesischer staatlicher Subventionen mit schädlichen Auswirkungen auf den EU-Binnenmarkt bieten.

Darüber hinaus werden gemäß Abschnitt 10(2) CAP Subventionen, die staatlichen chinesischen Unternehmen gewährt werden, automatisch als „spezifisch“ betrachtet - eine der gesetzlichen Voraussetzungen für die Auferlegung von Ausgleichszöllen im Rahmen des Übereinkommens über Subventionen und Ausgleichsmaßnahmen (C.IV). Außerdem verpflichtete sich China in einer ausdrücklichen Bestätigung von Artikel 25 des Übereinkommens über Subventionen und Ausgleichsmaßnahmen, spezifische Subventionen bis zum Zeitpunkt seines Beitritts zu notifizieren (Abschnitt 10.1 CAP) und alle Ausfuhrsubventionen und Importsubstitutionssubventionen zu beseitigen (Abschnitt 10.3 CAP).

16 Qin, JIEL 2004/4, S. 892.

17 Wolfgang Müller, in: Bungenberg et al. (Hrsg.), S. 219; S. https://archive.org/stream/Agre ementOnMarketAccess/ \#page/n3/mode/2up (11.03.2020). 
10. Subsidies ${ }^{18}$

1. China shall notify the WTO of any subsidy within the meaning of Article 1 of the Agreement on Subsidies and Countervailing Measures ("SCM Agreement"), granted or maintained in its territory, organized by specific product, including those subsidies defined in Article 3 of the SCM Agreement. The information provided should be as specific as possible, following the requirements of the questionnaire on subsidies as noted in Article 25 of the SCM Agreement.

2. For purposes of applying Articles 1.2 and 2 of the SCM Agreement, subsidies provided to state-owned enterprises will be viewed as specific if, inter alia, state-owned enterprises are the predominant recipients of such subsidies or state-owned enterprises receive disproportionately large amounts of such subsidies.

3. China shall eliminate all subsidy programmes falling within the scope of Article 3 of the SCM Agreement upon accession.

Schließlich enthält das CAP weitere allgemeine Verpflichtungen, u.a. in Bezug auf Transparenz (Abschnitt 2C), Staatshandel (Abschnitt 6), Preiskontrollen (Abschnitt 9) und Agrarsubventionen (Abschnitt 12). Dies wirft ein Licht auf die generelle Intention des CAP.

\section{Antisubventionsuntersuchungen der EU gegen China}

\section{Allgemeiner Überblick über die handelspolitischen Schutzinstrumente der EU}

Die EU verwendet ein besonderes System von Handelsschutzinstrumenten. Dazu gehören Antidumpingzölle (anti-dumping duties, ADDs), Ausgleichszölle gegen Subventionen sowie Schutzmaßnahmen (safeguard measures, SM) gegen dramatische Verschiebungen der Handelsströme. Diese Instrumente finden ihre Grundlage im WTO-Recht: Die WTO-rechtliche Grundlage für ihre Anwendung ist Artikel VI GATT 1947, das WTO-Anti-Dumping-Übereinkommen (ADA), das Übereinkommen über Subventionen und Ausgleichsmaßnahmen (ASCM) und das Übereinkommen über Schutzmaßnahmen (SGA). ${ }^{19}$ In Bezug auf den Handel mit China sind auch die spezifischen Bestimmungen des CAP Teil des geltenden Rechtsrahmens. Abschließend ist zu beachten, dass die Ad Note zu Artikel VI:1 GATT vorsieht, dass in Antidumping-Angelegenheiten die Inlandspreise ignoriert werden können in "a country which has a complete or substantially complete monopoly of its trade and where domestic prices are fixed by the State" in einem solchen Umfang, dass "a strict comparison with domestic prices in such a country may not always be appropriate."

18 Abschnitt 10 CAP.

19 Agreement on Implementation of Article VI of the General Agreement on Tariffs and Trade 1994, 15.04.1994; Agreement on Subsidies and Countervailing Measures, 15.04.1994; and Agreement on Safeguards, 15.04.1994. 
In der EU werden diese WTO-Regeln durch Verordnungen in EU-Recht umgesetzt; die Anti-Dumping-Verordnung ${ }^{20}$ und die Antisubventionsverordnung ${ }^{21}$ er- $^{-}$ möglichen es der EU, ihre Produzenten vor Verzerrungen des internationalen Wettbewerbs infolge gedumpter oder subventionierter Einfuhren zu schützen. Insofern sollen insbesondere Antidumpingzölle und Ausgleichszölle unter anderem den künstlichen Vorteilen entgegenwirken, von denen Produkte aus Drittländern im internationalen Handel durch staatlich bewirkte oder privat organisierte Verzerrungen profitieren können. Darüber hinaus kann die EU mit der Verabschiedung von Schutzmaßnahmen auf Grundlage der Einfuhrverordnung reagieren. ${ }^{22}$

\section{Handelsschutzinstrumente und staatlich verzerrte Märkte}

Heute ist China nach den Vereinigten Staaten der zweitwichtigste Handelspartner der EU, mit Importen in Höhe von fast 400 Milliarden US-Dollar im Jahr 2018. Die europäische Wirtschaft sieht sich durch solche massiven Importe aus China in die EU unter Druck gesetzt. Handelsschutzinstrumente sollen den „unfairen“ Einfluss staatlich unterstützter chinesischer Waren, die in die EU importiert werden, beheben. So liegt der Zweck der Antisubventionsverordnung im Ausgleich von Subventionen, die direkt oder indirekt für die Herstellung, Produktion, Ausfuhr oder den Transport von Produkten aus einem Nicht-EU-Land gewährt werden und deren Vertrieb auf dem EU-Markt eine Schädigung der europäischen Wirtschaft verursacht (Artikel 1 Abs. 1 Antisubventionsverordnung).

China ist das Land, welches bislang am häufigsten Ziel von Antidumping- und Antisubventionsuntersuchungen der EU geworden ist; in den letzten zwanzig Jahren hat die EU-Kommission ihre Untersuchungen im Zusammenhang mit einer möglichen Anwendung von handelspolitischen Schutzinstrumenten weitgehend auf chinesische Einfuhren konzentriert. Bislang ist hier das vorherrschende Instrument das der Antidumping-Untersuchungsverfahren und -zölle gewesen. ${ }^{23}$

Im Jahr 2017 hat die EU die Antidumpingverordnung im Hinblick auf Importe von staatlich verzerrten Märkten durch die Einführung neuer Berechnungsmethoden für

20 VO (EU) 2016/1036, über den Schutz gegen gedumpte Einfuhren aus nicht zur Europäischen Union gehörenden Ländern (kodifizierter Text), ABl. L 176 v. 30.06.2016, S. 21. Kodifizierte Fassung abrufbar unter: https://eur-lex.europa.eu/legalcontent/EN/TXT/PDF/? uri=CELEX:02016R1036-20180608\&from=EN (11.03.2020).

21 VO (EU) 2016/1037, über den Schutz gegen subventionierte Einfuhren aus nicht zur Europäischen Union gehörenden Ländern (kodifizierter Text), ABl. L 176 v. 30.06.2016, S. 55. Kodifizierte Fassung abrufbar unter: https://eur-lex.europa.eu/legal-content/EN/TXT/P DF/?uri=CELEX:02016R1037-20180608\&from=EN (11.03.2020).

22 VO (EU) 2015/478, über eine gemeinsame Einfuhrregelung, ABl.L 83 v. 27.03.2015, S. 16-33.

23 Für einen Überblick zu Handelsschutzmaßnahmen in 2019, s. Europäische Kommission, Anti-dumping, Anti-subsidy, Safeguard. Statistics covering 2019, Dezember 2019, abrufbar unter: https://trade.ec.europa.eu/doclib/docs/2020/january/tradoc_158564.pdf (11.03.2020). Für einen Überblick zu laufenden Untersuchungen, s. https://trade.ec.europ a.eu/tdi/ (11.03.2020). 
den Normalwert von Waren geändert. ${ }^{24}$ Es muss dafür zunächst nachgewiesen werden, dass „nennenswerte Verzerrungen“ in der Wirtschaft des Exportlandes infolge staatlicher Eingriffe bestehen. ${ }^{25} \mathrm{Zu}$ diesem Zweck prüft die Kommission alle im Rahmen einer Untersuchung vorgelegten Beweise, die auch von Seiten der EU-Industrie eingebracht werden können. In diesem Zusammenhang kann die Kommission Berichte erstellen. ${ }^{26}$ Der zu China veröffentlichte Bericht beleuchtet bestimmte Aspekte der chinesischen Wirtschaft und ist der Gesamtwirtschaft des Landes, den wichtigsten Produktionsfaktoren, die in allen Herstellungsprozessen eingesetzt werden, sowie bestimmten Wirtschaftssektoren gewidmet. Nach Aufgabe der „traditionellen“ Drittland-Normalwertberechnungsmethode werden dennoch niedrigere Dumpingspannen bei Einfuhren aus China erwartet - dies als Folge der schwierigeren und zeitintensiveren Untersuchungen (C.IV). Daher wird eingewendet, dass die Änderung der EU-Antidumpingverordnung die Wirksamkeit der handelspolitischen Schutzinstrumente der EU verringert habe, was sich negativ auf die EU-Industrie auswirke. ${ }^{27}$ Eine mögliche Abhilfe, um diesem Effekt entgegenzuwirken und um einen effektiven Handelsschutz beizubehalten, könnte in der verstärkten Anwendung von Ausgleichszöllen liegen.

\section{Aktueller Einsatz von Antisubventionsuntersuchungen gegen China}

Das bestehende EU-Recht erlaubt Antisubventionsuntersuchungen, die die EU-Industrie vor subventionierten Importen aus China schützen. ${ }^{28}$ Das Thema der staatlich verzerrten Märkte hat allerdings nur bei der Reform der Anti-Dumping-Verordnung viel Aufmerksamkeit erfahren, hingegen wenig bei der jüngsten Änderung der Antisubventionsverordnung. Im EU-Kontext stellt sich die Frage, ob die Antisubventionsverordnung einen angemessenen Schutz gegen Subventionierungen von Unternehmen in Drittländern bietet, die eine verzerrende Wirkung auf die europäische Wirtschaft im Allgemeinen oder für bestimmte Sektoren haben.

24 S. VO (EU) 2017/2321, zur Änderung der Verordnung (EU) 2016/1036 über den Schutz gegen gedumpte Einfuhren aus nicht zur Europäischen Union gehörenden Ländern und der Verordnung (EU) 2016/1037 über den Schutz gegen subventionierte Einfuhren aus nicht zur Europäischen Union gehörenden Ländern, ABl. L 338 v. 19.12.2017, S. 1. Vgl. Hierzu insbesondere den Beitrag von T. Müller-Ibold, in diesem Heft, S. $239 \mathrm{ff}$.

25 S. neuen Artikel 2(6a) Antidumpingverordnung.

26 Commission Staff Working Report on significant distortions in the economy of the People's Republic of China for the purposed of trade defence investigations, 20.12.2017, SWD(2017) 483 final/2. Die Kommission kündigte ähnliche Maßnahmen mit Bezug zu Russland an; bisher wurde jedoch kein Report veröffentlicht.

27 Europäische Kommission, Open public consultation regarding the possible change in the methodology to establish dumping/subsidisation in trade defence investigations concerning the Peoples' [sic] Republic of China, abrufbar unter: http://trade.ec.europa.eu/doclib/doc s/2016/february/tradoc_154258.pdf (11.03.2020), S. 2; für mehr Informationen s. http://tr ade.ec.europa.eu/consultations/index.cfm?consul_id=191 (11.03.2020). Die Befragung wurde geschlossen am 20.04.2016.

28 Dazu beispielsweise Vermulst/Gatta, WTR 2012/3, S. 527-553. 
In den letzten zehn Jahren wurden in den meisten westlichen Industrieländern verstärkt Antisubventionsuntersuchungen durchgeführt. ${ }^{29}$ Bis 2006 hatten die USA von der Durchführung von Antisubventionsverfahren gegen Länder ohne Marktwirtschaft (non-market economies, NMEs) abgesehen, ${ }^{30}$ kamen aber 2007 zu dem Schluss, dass Chinas Wirtschaft nunmehr ausreichend liberalisiert sei und daher die Anwendung von Antisubventionsmaßnahmen erlaubt sei. ${ }^{31}$ Andere WTO-Mitgliedstaaten, darunter auch die EU, folgten diesem Ansatz bald. Die ersten EU-Untersuchungen gegen chinesische Importe begannen 2010.32

Ungeachtet der Tatsache, dass die Anwendung des Antisubventions-Abkommens der WTO wie auch der EU-Antisubventionsverordnung verfahrensrechtlich schwierig ist (IV), wurden bereits Ausgleichszölle von 35,9 \% auf warmgewalzte Flachstahlprodukte bzw. von 51,08 \% auf Reifen aus China erlassen. ${ }^{33}$ Die EU hat bisher zwölf Antisubventionsverfahren gegen China eingeleitet, von denen acht zur Einführung eines endgültigen Ausgleichszolls geführt haben; zwei Verfahren sind derzeit noch anhängig. ${ }^{34}$ In den bisherigen Verfahren ging es um Subventionen in Bezug auf Rohstoffe, Landnutzungsrechte, Wasser und Elektrizität sowie um Steuervergünstigungen. ${ }^{35} \mathrm{Zu}$ den betroffenen Sektoren gehören Holz und Papier, Eisen und Stahl, Elektronikartikel, Fahrräder und erneuerbare Energien. ${ }^{36}$ Im Vergleich dazu haben die USA eine umfassendere Praxis der strikten Durchsetzung ihrer Ausgleichszollgesetzgebung entwickelt, um unlauteres Handelsverhalten durch chinesische Subventionen zu unterbinden. Die USA haben in der Vergangenheit mehr als 50 Antisubventions-

29 S. Chiang, GJIL 2017/2, S. $853 \mathrm{ff}$.

30 Eine Politik, die seit dem Fall Georgetown Steel von 1986 beibehalten wurde. See Georgetown Steel Corporation, et al. v. United States, 801 F.2d 1308, 8 ITRD 1161, 4 Fed. Cir. (T) $143,18.09 .1986$.

31 US Department of Commerce, Cases C-570-959 and A-570-958, Coated Paper Suitable for High-Quality Print Graphics Using Sheet-Fed Presses (initiiert 20.10.2009), abrufbar unter: http://enforcement.trade.gov/stats/inv-initiations-2000-current.html, A-570-958 (11.03.2020). Die Untersuchung schloss wegen mangelnder Schädigung ohne endgültigen Erlass von Ausgleichszöllen ab. S. US International Trade Commission, Coated Free Sheet Paper From China, Indonesia, and Korea, 13.12.2007, 72 Fed. Reg. 70,892, Investigation No. 701-TA-444-446 (Final) and 731-TA-1107-1109 (Final).

32 DVO (EU) 452/2011, zur Einführung eines endgültigen Ausgleichszolls auf die Einfuhren von gestrichenem Feinpapier mit Ursprung in der Volksrepublik China, ABl. L 128 v. 14.05.2011, S. $18 \mathrm{ff}$.

33 Europäische Kommission, 37th Annual Report from the Commission to the Council and the European Parliament on the EU's Anti-Dumping, Anti-Subsidy and Safeguard activities and the Use of trade defence instruments by Third Countries targeting the EU in 2018, 27.03.2019, $\mathrm{COM}(2019) 158$ final, S. 9.

34 S. Annex 1.

35 S., z.B., DVO (EU) Nr. 215/2013, zur Einführung eines Ausgleichszolls auf die Einfuhren bestimmter organisch beschichteter Stahlerzeugnisse mit Ursprung in der Volksrepublik China, ABl. L 73 v. 15.3.2013, S. 16-97.

36 Basiert auf Europäische Kommission, Anti-subsidies investigations database, 30.01.2019, abrufbar unter: https://trade.ec.europa.eu/doclib/html/157607.htm (11.03.2020). 
maßnahmen gegen China (bis Ende 2019) ${ }^{37}$ in einer Vielzahl von Sektoren verhängt. ${ }^{38}$

\section{Allgemeine Durchführung von EU-AS-Untersuchungen}

Von dem Vorliegen einer Subvention, die Gegenstand von Ausgleichsmaßnahmen sein kann, wird ausgegangen, wenn:

- eine Regierung im Ursprungs- oder Ausfuhrland eine finanzielle Beihilfe leistet oder irgendeine Form der Einkommens- oder Preisstützung besteht ${ }^{39}$ (Art. 3 Abs. 1 Antisubventionsverordnung),

- dadurch ein Vorteil gewährt wird (Art. 3 Abs. 2 Antisubventionsverordnung),

- diese „Subvention“ spezifisch für ein Unternehmen oder einen Wirtschaftszweig oder eine Gruppe von Unternehmen oder Wirtschaftszweigen ist (Art. 4 Antisubventionsverordnung),

- eine Schädigung der EU-Wirtschaft festgestellt wird (Art. 8 Antisubventionsverordnung),

- ein kausaler Zusammenhang zwischen der Schädigung und den subventionierten Einfuhren besteht (Art. 8 Antisubventionsverordnung), und

- die Einführung von Maßnahmen dem Interesse der Union nicht zuwiderläuft (Art. 31 Antisubventionsverordnung).

Nur wenn alle diese Bedingungen erfüllt sind, kann die Kommission Ausgleichsmaßnahmen einführen.

Das Untersuchungsverfahren beginnt mit einem offiziellen Antrag (Art. 10 Antisubventionsverordnung). Bereits in diesem Stadium muss der Antragsteller ausreichende Beweise für Subventionen verfügen, die der Einfuhr einer fraglichen Ware zugutekommen. Nach der Einleitung des Verfahrens versendet die Kommission Fragebögen an alle interessierten Parteien (Art. 11 Antisubventionsverordnung), einschließlich der Regierung des Ausfuhrlandes, der Ausführer der fraglichen Ware und der EU-Hersteller. Parteien, die den Fragebogen nicht beantworten, werden als nicht an der Untersuchung mitarbeitend betrachtet. Die Kommission wird die Untersuchung ungeachtet der Nichtmitwirkung einzelner Parteien grundsätzlich fortsetzen und kann sich dabei auf andere verfügbare Informationen stützen (Art. 29 Antisubventionsverordnung). Für einen nicht kooperierenden Ausführer dürfte der Zoll in der Regel höher sein, als wenn er kooperiert hätte.

Die zentralen Schritte des Antisubventionsuntersuchungsverfahrens der EU sind von strengen Zeitvorgaben geleitet. ${ }^{40}$ Innerhalb von 45 Tagen nach der Einreichung eines Antrags wird eine Bekanntmachung über die Einleitung des Verfahrens (notice

37 S. https://enforcement.trade.gov/frn/summary/prc/prc-fr.htm (31.01.2020).

38 S. United States Trade Representative, 2018 Report to Congress On China's WTO Compliance, Februar 2019, S. 79, abrufbar unter: https://ustr.gov/sites/default/files/2018-UST R-Report-to-Congress-on-China\%27s-WTO-Compliance.pdf (11.03.2020).

39 Im Sinne des Art. XVI General Agreement on Tariffs and Trade 1994 (GATT 1994).

40 S. Annex 2. 
of initiation) veröffentlicht. Stellt die Europäische Kommission innerhalb von neun Monaten nach der Einleitungsbekanntmachung Beweise für eine schadensverursachende Subventionierung fest, können vorläufige Maßnahmen mit einer Höchstdauer von vier Monaten eingeführt werden (Art. 12 Antisubventionsverordnung). Endgültige Ausgleichszölle müssen innerhalb von 13 Monaten nach der Einleitung des Verfahrens eingeführt werden (Art. 15 Antisubventionsverordnung).

\section{Praktische Schwierigkeiten bei China betreffenden Antisubventionsuntersuchungen}

Die herkömmliche Antisubventionsuntersuchung ist während der gesamten Untersuchung zahlreicher praktischer Schwierigkeiten und Hürden ausgesetzt. Insbesondere in Bezug auf Subventionen in China, an denen Staatsunternehmen beteiligt sind, ergeben sich Probleme durch die mangelnde Transparenz dieser Unternehmen; der ihnen gewährten Subventionsregelungen; der Frage, ob diese Unternehmen als öffentliche Körperschaften im Sinne des Anwendungsbereichs des Übereinkommens über Subventionen und Ausgleichsmaßnahmen eingestuft werden können; sowie der Anwendung einer funktionierenden und wirksamen Berechnungsmethode, die es erlaubt, die chinesischen Inlandspreise außer Acht zu lassen.

\section{Mangel an Transparenz}

Das Übereinkommen über Subventionen und Ausgleichsmaßnahmen verlangt von den WTO-Mitgliedern gemäß seines Artikel 25.2, bestimmte Subventionen zu notifizieren, die in ihrem Hoheitsgebiet gewährt oder aufrechterhalten werden. Viele WTO Mitgliedstaaten haben diese Verpflichtung völlig ignoriert oder sind bei der Bereitstellung der erforderlichen Notifikationen ihrer Subventionen säumig. ${ }^{41} 78$ WTO-Mitglieder (48\%) hatten noch nicht die in 2017 fälligen Subventionen notifiziert, 63 Mitglieder (38\%) die in 2015 und 56 Mitglieder (34\%) ihre in 2013 fälligen Notifikationen noch nicht eingereicht. ${ }^{42}$ China hat seit 2006 einige unvollständige Notifizierungen vorgenommen und nur in begrenztem Umfang Subventionen von lokalen Behörden und staatlichen Banken beigefügt.

Jedes Mitglied kann in einer so genannten Gegennotifikation (counter-notification; Artikel 25.10 des Übereinkommens) Fragen an den SCM-Ausschuss richten. Davon abgesehen sind jedoch keine Konsequenzen für die Unterlassung der Notifikation von Subventionen und Subventionsprogrammen vorgesehen; auch wurden bislang keine Streitigkeiten wegen dieses Verstoßes gegen das Übereinkommen eingeleitet.

Abgesehen von der allgemeinen Frage nach Konsequenzen der Nichtnotifizierung von Subventionen, beeinträchtigt der Mangel an Transparenz auch die Möglichkeiten der Kommission in Untersuchungsverfahren, eine Analyse u.a. der Erfüllung der Tatbestandsvoraussetzungen der öffentlichen Körperschaft abzuschließen, zu begründen, dass die untersuchte Subvention eine bedeutende Schädigung verursacht, sowie

41 Bown/Hillman, JIEL 2019/4, S. 570.

42 WTO Committee on Subsidies and Countervailing Measures Meeting, 23.10.2018. 
den Nachweis zu erbringen, dass Schädigungen durch die jeweiligen untersuchten Begünstigungen und nicht durch andere Faktoren verursacht wurden.

\section{Ungeeignete Definition der öffentlichen Körperschaft}

Das Berufungsgremium (Appellate Body, AB) hat den Begriff „öffentliche Körperschaft" eingegrenzt, um nur diejenigen Körperschaften zu erfassen, die Regierungsfunktionen ausüben. ${ }^{43}$ Deshalb entziehen sich viele Staatsunternehmen dem Anwendungsbereich des Art.1.1 lit.a) Abs. 1 Ziff.iv) des Übereinkommens über Subventionen und Ausgleichsmaßnahmen. ${ }^{44}$ Die Rolle von Staatsunternehmen als subventionsvergebende Einrichtungen ist im Übereinkommen damit nicht ausreichend geregelt. Darüber hinaus ist es für eine Untersuchungsbehörde nicht leicht, konkrete und umfassende Beweise für die Kontrolle der Regierung über ein staatliches Unternehmen vorzulegen. ${ }^{45}$ Bown und Hillman weisen auf Schwierigkeiten „in Bezug auf (i) den Nachweis der Kontrolle der Regierung über ein Unternehmen als Teil des Nachweises, dass dieses Unternehmen möglicherweise ein „Subventionsgeber“ ist, hin, sowie (ii) den Nachweis, dass Maßnahmen eines privaten Unternehmens auf „Anweisung“ der Regierung durchgeführt wurden [...]“.46 Dies gilt insbesondere dann, wenn die Subventionen in intransparenten Volkswirtschaften wie China gewährt werden, wo die Dokumentation von Regierungsmaßnahmen gegenüber Staatsunternehmen nicht zu den öffentlichen Aufgaben gehört. Folglich muss sich die Kommission auf die jeweiligen gerade verfügbaren Fakten stützen, wenn das untersuchte Land keine Informationen liefert oder die Untersuchung behindert. Nichtsdestotrotz hat die Kommission staatliche Stahlproduzenten, staatliche Banken und staatliche Versicherungsgesellschaften als öffentliche Körperschaften qualifiziert. ${ }^{47}$

\section{Fehlen einer effektiven Berechnungsmethode}

Die Höhe der fraglichen Subventionen wird grundsätzlich anhand des dem Empfänger im Untersuchungszeitraum gewährten Vorteils berechnet (Art. 14 des Übereinkommens über Subventionen und Ausgleichsmaßnahmen). In der Praxis ist es schwierig, die Umstände festzulegen, unter denen Inlandspreise abgelehnt werden können und wie dann ein angemessener Bewertungsmaßstab festgelegt werden soll, sowie wann und in welchem Umfang Preise außerhalb des Marktes des subventionierenden Mitglieds verwendet werden können. ${ }^{48}$ Artikel 6(d) Antisubventionsverordnung erlaubt es, auf alternative Preise zurückzugreifen, wenn es im Exportland keine Marktpreise gibt. Wenn keine Marktbedingungen existieren, die als geeignete Bemessungsgrund-

43 Appellate Body Report, United States - Definitive Anti-Dumping and Countervailing Duties on Certain Products from China, WT/DS379/AB/R, 25.03.2011.

44 EU concept paper on WTO reform, Juli 2018, abrufbar unter: http://trade.ec.europa.eu/d oclib/docs/2018/september/tradoc_157331.pdf (11.03.2020).

45 Chu/Lee, JWT 2019/6, S. 917 f.

46 Bown/Hillman, JIEL 2019/4, S. 569 (von den Autoren übersetzt).

47 Van Bael E Bellis, S. $631 \mathrm{f}$.

48 Joint Statement of the Trilateral Meeting of the Trade Ministers of Japan, the United States and the European Union, Washington, D.C., 14.01.2020, abrufbar unter: https://trade.ec.e uropa.eu/doclib/docs/2020/january/tradoc_158567.pdf (11.03.2020), para. 5. 
lage herangezogen werden können, werden die in dem Land herrschenden Bedingungen:

- „um einen angemessenen Parameter“ angepasst, um den normalen Marktbedingungen Rechnung zu tragen, oder

- durch die „auf dem Markt eines anderen Landes oder auf dem Weltmarkt herrschenden Bedingungen" ersetzt. ${ }^{49}$

Dabei wenden die Behörden im Allgemeinen drei verschiedene Methoden an: Anpassung der bestehenden Preise im Land an die Marktbedingungen, Konstruktion eines alternativen Preises auf der Grundlage von Durchschnittsdaten oder Verwendung von Preisen auf einem Drittmarkt. ${ }^{50}$ Die Möglichkeit einer Abweichung von den Inlandspreisen wurde bereits vom WTO-Berufungsgremium in der Sache Softwood Lumber $I V^{51}$ akzeptiert. In späteren Entscheidungen wurde dieser Ansatz bestätigt und erweitert. In $U S-A D D$ and $C V D$, zum Beispiel, entschied das Berufungsgremium und bestätigte damit das Panel -, dass Artikel 14(b) des Übereinkommens die Möglichkeit bietet, die Zinssätze in China als Bemessungsgrundlage für Darlehen für staatliche Banken außer Acht zu lassen..$^{52}$ Obwohl das Berufungsgremium bereits angedeutet hat, dass ähnliche Überlegungen auch für die anderen Absätze von Artikel 14 des Übereinkommens gelten könnten, wurden bislang in dieser Hinsicht keine Entscheidungen verabschiedet ${ }^{53}$ in der EU wurde bislang keine nachträgliche Änderung von Artikel 6 der Antisubventionsverordnung vorgenommen, um diese Rechtsprechung des Schiedsgerichts einzuarbeiten.

Auch bei den Berechnungsmethoden beeinflusst ein Mangel an Transparenz die Möglichkeiten der Untersuchungsbehörde, nachzuweisen, ob ein Vorteil durch einen finanziellen Beitrag gewährt wird, indem Gelder oder Ressourcen zu unter dem Marktpreis liegenden Preisen gewährt werden.

\section{Andere (allgemeine) Probleme bei Antisubventionsuntersuchungen}

Darüber hinaus werden als allgemeineres Problem des derzeitigen Antisubventionsregimes die Abhilfemaßnahmen als unzureichend angesehen. Ausgleichszölle sind nur dann möglich, wenn die subventionierten Waren in ein Land importiert werden, das über eine einheimische Industrie verfügt, die ähnliche Produkte herstellt und nachweisen kann, dass sie durch die subventionierten Einfuhren geschädigt wird. Unlauterer Wettbewerb durch subventionierte Produkte auf Drittlandsmärkten ist nicht erfasst. ${ }^{54}$ Darüber hinaus können Ausgleichszölle zu mehr (unlauterem) Wettbewerb auf Drittmärkten führen.

49 Art. 6(d) Antisubventionsverordnung.

50 Kelly, GEJ 2011/2, S. $11 \mathrm{ff}$.

51 Appellate Body Report, United States - Final Countervailing Duty Investigation with Respect to Certain Softwood Lumber from Canada, WT/DS257/AB/R, 17.02.2004, paras $167 \mathrm{f}$.

52 Appellate Body Report, United States - Definitive Anti-Dumping and Countervailing Duties on Certain Products from China, WT/DS379/AB/R, 25.03.2011, paras 484 und $488 \mathrm{f}$.

53 S. Appellate Body Report, United States - Definitive Anti-Dumping and Countervailing Duties on Certain Products from China, WT/DS379/AB/R, 25.03.2011, para. 10.122.

54 Bown/Hillman, JIEL 2019/4, S. 571. 
Ausgleichszölle erfordern eine umfangreiche und damit (zu) lange Untersuchung, insbesondere um Daten zu sammeln und die Subventionen zu untersuchen. Es kann mehrere Jahre dauern, bis ein Beschwerdeführer eine Subventionsanfechtung vorbringt und hiermit auch erfolgreich ist.

Die derzeitigen EU-Vorschriften erfassen auch nicht die am stärksten handelsverzerrenden Arten von Subventionen, die zu Überkapazitäten beitragen und dadurch den internationalen Handel stark verzerren. ${ }^{55}$

Darüber hinaus fallen „andere“ staatliche Praktiken, die den Effekt einer Subvention haben - wie die unterschiedliche Anwendung von Exportsteuern und die unterschiedliche Rückerstattung von Mehrwertsteuern in der industriellen Lieferkette - nicht unter die Definition einer Subvention. ${ }^{56}$ Für EU-Unternehmen besteht zudem ein „Nachteil“ darin, dass das EU-Beihilferecht nur auf staatliche Fördermaßnahmen der EU-Mitgliedstaaten anwendbar ist und sich nicht auf Subventionen durch Drittstaaten erstreckt.

\section{E. Erste Ideen für neue Antisubventionsuntersuchungsmethoden bei Chinesischen Importen}

Das Antisubventions- und Ausgleichszollregime könnte in Zukunft auf die chinesischen Importe ausgeweitet werden. Abschnitt 15(b) CAP wird weitgehend übersehen, bietet aber bei näherer Betrachtung „ungeahnte Möglichkeiten“, insbesondere bei chinesischen Importen, China-spezifische alternative Vorgehensweisen zur Bestimmung der anfechtbaren Begünstigungen auszuarbeiten (E.I). Abgesehen der Ansätze, die wohl von einem Rückgriff auf Abschnitt 15(b) CAP erfasst sind, werden nachfolgend auch andere Vorschläge angeführt, die möglicherweise nicht durch das CAP abgedeckt sind, aber dennoch den Verlauf von Antisubventionsuntersuchungen gegen China effizienter machen würden (E.II).

\section{Anwendung von Abschnitt 15(b) CAP, kombiniert mit einer Verlagerung der Beweislast}

Abschnitt 15(b) CAP lautet:

(b) In proceedings under Parts II, III and V of the SCM Agreement, when addressing subsidies described in Articles 14(a), 14(b), 14(c) and 14(d), relevant provisions of the SCM Agreement shall apply; however, if there are special difficulties in that application, the importing WTO Member may then use methodologies for identifying and measuring the subsidy benefit which take into account the possibility that prevailing terms and conditions in China may not always be available as appropriate benchmarks. In applying such

55 Europäische Kommission, 37th Annual Report from the Commission to the Council and the European Parliament on the EU's Anti-Dumping, Anti-Subsidy and Safeguard activities and the Use of trade defence instruments by Third Countries targeting the EU in 2018, 27.03.2019, COM(2019) 158 final, Annex 8. S. auch Bhala/Kim, AJWH 2019/1, S. 1-32.

56 Bown/Hillman, JIEL 2019/4, S. 558. 
methodologies, where practicable, the importing WTO Member should adjust such prevailing terms and conditions before considering the use of terms and conditions prevailing outside China.

So erlaubt Abschnitt 15(b) CAP die Verwendung alternativer Berechnungsgrundlagen der Höhe einer vom chinesischen Staat gewährten Subvention, wenn die Untersuchungsbehörden bei der Anwendung der Unterabsätze von Artikel 14 des Übereinkommens über Subventionen und Ausgleichsmaßnahmen auf besondere Schwierigkeiten stoßen. Abschnitt 15(b) CAP ermöglicht es einem einführenden WTOMitglied, im Falle einer chinesischen Subvention alternative Bemessungsgrundlagen zu verwenden, ohne die Anforderungen von Artikel 14 des Übereinkommens in der Auslegung der bestehenden WTO-Rechtsprechung erfüllen zu müssen. Es ist anscheinend von dieser Möglichkeit in der Vergangenheit bisher kein Gebrauch gemacht worden.

Um auf diese Bestimmung zurückgreifen zu können, müssen die folgenden Punkte berücksichtigt werden: Abschnitt 15(b) CAP enthält eine Tatbestandsvoraussetzung (bei „besonderen Schwierigkeiten“), sowie als „Rechtsfolge“ die Möglichkeiten von Anpassungen sowie der Verwendung alternativer Berechnungsmethoden. Der hier nachfolgend unterbreitete Vorschlag kombiniert diese Punkte jeweils mit einer Verlagerung der Beweislast; es obliegt China, den Nachweis der herrschenden Marktwirtschaftsbedingungen zu erbringen, damit ansonsten mögliche alternative Berechnungsgrundlagen nicht angewendet werden. Damit erfolgt mit diesem Vorschlag eine Auflösung einiger der drängendsten praktischen Schwierigkeiten in den laufenden Antisubventionsuntersuchungen, wie oben (D) dargelegt, einschließlich der mangelnden Transparenz und der Probleme beim Nachweis der Existenz einer öffentlichen Körperschaft.

\section{Die Bedingung: „Besondere Schwierigkeiten“}

Bei der Untersuchung von Importen aus China kann sich die EU bei der Anwendung des Verfahrens nach Artikel 14 des Übereinkommens über Subventionen und Ausgleichsmaßnahmen auf „besondere Schwierigkeiten“ berufen, um sich auf Abschnitt 15(b) CAP zu beziehen. Diese „besonderen Schwierigkeiten“ sind bisher nicht definiert worden. ${ }^{57}$ Solange es keine Entscheidung des Berufungsgremiums zu dieser Frage gibt, hängt die Definition dieser Voraussetzung von den untersuchenden Behörden ab. ${ }^{58}$ Klar ist, dass die Anforderungen niedriger sein sollten als die für die Anwendung von Artikel 14 des Übereinkommens, der sorgfältig ausgearbeitete und vom Berufungsgremium interpretierte Bedingungen enthält. ${ }^{59}$

Die EU-Kommission könnte hier argumentieren, dass diese besonderen Schwierigkeiten im Falle von (staatlich verzerrten Märkten in) China grundsätzlich bestehen. Dies wäre ein Ansatz, der dem Anti-Dumping-Rechtsrahmen des neuen Artikels 2

57 Müller, Alternative Benchmarks, S. $77 \mathrm{ff}$.

58 Sophia Müller, in Bungenberg et al. (Hrsg.), S. 133.

59 Zhou/Goa/Bai, ICLQ 2019, S. 1014 ff. 
Absatz 6a Antidumpingverordnung vergleichbar ist. Die Kommission hat dort ausführlich dargelegt, warum der chinesische Markt stark „staatlich verzerrt“ ist. ${ }^{60} \mathrm{Chi}^{-}$ nesische Exporteure können dann im Einzelfall darlegen, dass sie keine staatlichen Subventionen erhalten, wenn sie ohne Beschränkungen in der EU Geschäfte machen wollen. ${ }^{61}$ Es wäre auch möglich, es der chinesischen Regierung zu überlassen, ein transparentes System staatlicher Subventionen zu schaffen, dies auch ggf. in einem sektorspezifischen Ansatz, um zu widerlegen, dass gerade keine besonderen Schwierigkeiten vorliegen.

Alternativ könnte bereits die Präsenz von Staatsunternehmen auf einem Markt, in einem spezifischen Wirtschaftssektor oder sogar in der Produktionskette einer importierten Ware, die Gegenstand der Untersuchung ist, ein bestimmendes Indiz für das Vorliegen besonderer Schwierigkeiten sein. Chinesische Exporteure müssten dann Informationen über das Nichtvorliegen staatlicher Einflussnahme auf staatliche Unternehmen darlegen.

Informationen über chinesische Subventionen fehlen oft, sind unzureichend oder aus anderen Gründen schwer zu erhalten (s.o.). Die Schwelle für besondere Schwierigkeiten könnte auch so ausgestaltet werden, dass man Situationen einbezieht, in denen ein offensichtlicher Mangel an Informationen besteht oder in denen die Einholung von Informationen schwierig ist. ${ }^{62}$ Abschnitt 15(b) CAP könnte daher so lange herangezogen werden, bis volle Transparenz über die Subventionsprogramme erreicht ist und China in ausreichender Weise an der jeweiligen Antisubventionsuntersuchung mitarbeitet.

Der Vorteil dieser Interpretation von „besonderen Schwierigkeiten“ liegt darin, dass diese Tatbestandsvoraussetzung der besonderen Schwierigkeiten in Abschnitt 15(b) CAP mit anderen Gesichtspunkten verknüpft werden kann. Abschnitt 15(b) CAP könnte den Untersuchungsbehörden der Einfuhrländer erlauben, alternative Methoden zur Ermittlung und Berechnung von Begünstigungen anzuwenden, bis China bestimmte Schwierigkeiten angeht oder zumindest den Beweis erbringt, dass diese Schwierigkeiten im konkreten Fall nicht vorliegen. Daher bietet Abschnitt 15(b) CAP mehr Möglichkeiten als Artikel 14 des WTO-Übereinkommens oder Artikel 6 Antisubventionsverordnung, indem er weitere Ansätze für eine Lösung anderer Probleme eröffnet, einschließlich der (Neu-)Definition der öffentlichen Körperschaft und des Mangels an öffentlich zugänglichen Informationen.

60 Commission Staff Working Report on significant distortions in the economy of the People's Republic of China for the purposed of trade defence investigations, 20.12.2017, SWD(2017) 483 final $/ 2$.

61 S. beispielsweise Joint Statement of the Trilateral Meeting of the Trade Ministers of Japan, the United States and the European Union, Washington, D.C., 14.01.2020, para. 2; Bundesverband der Deutschen Industrie, BDI Policy Papers China, Januar 2019.

62 Zhou/Goa/Bai, ICLQ 2019, S. 1014-1017. 


\section{Anpassungen der Bemessungsgrundlagen}

Wenn die Kommission feststellt, dass besondere Schwierigkeiten bei der Anwendung von Artikel 14 des Übereinkommens über Subventionen und Ausgleichsmaßnahmen bestehen und China nicht in der Lage war, das Gegenteil zu beweisen, erlaubt Abschnitt 15(b) CAP dem importierenden WTO-Mitglied, die chinesischen „vorherrschenden Bedingungen“ anzupassen. Hier bieten sich neue Möglichkeiten:

Eine solche Anpassung könnte z.B. in der Anwendung einer automatischen (z.B.) $20 \%$-Regelung liegen. Bei dieser Option könnte die EU argumentieren, dass jede Ware, die in einem staatlich verzerrten Markt produziert wird, per se subventioniert ist, und der Vorteil beispielsweise bei $20 \%$ des Wertes der importierten Ware liegt. Es kann hier nur ein Prozentsatz definiert werden, der ein relevantes Niveau des Vorteils auf der Grundlage des Ausmaßes der staatlichen Verzerrung widerspiegelt. Dieser Prozentsatz kann entweder auf Länderebene festgelegt oder differenziert für unterschiedliche chinesische Sektoren ausgestattet werden.

Diese Anpassungsmethode würde sich an den Entwicklungen im Bereich des öffentlichen Beschaffungswesens orientieren, wo die EU seit 2012 das Internationale Beschaffungsinstrument (International Procurement Instrument, IPI) diskutiert. ${ }^{63}$ Die Kommission hat am 29. Januar 2016 einen überarbeiteten Vorschlag vorgelegt. ${ }^{64}$ In Fällen von angeblicher Diskriminierung von EU-Unternehmen auf ausländischen Beschaffungsmärkten durch ein Drittland leitet die Kommission eine öffentliche Untersuchung ein. In Fällen diskriminierender Beschränkungen gegenüber Waren, Dienstleistungen und/oder Lieferanten aus der EU würde die Kommission das betreffende Land zu einer Konsultation über die Öffnung seines Beschaffungsmarktes einladen. Schließlich kann die Kommission nach Rücksprache mit den Mitgliedstaaten eine „Preisstrafe“ auf Angebote aus dem Zielland mit einem Gesamtwert von mindestens 5 Mio. EUR anwenden, die zu mindestens $50 \%$ aus Waren und Dienstleistungen aus dem Zielland bestehen. Bei Angeboten aus diesem Land wird davon ausgegangen, dass sie einen von bis zu $20 \%$ des tatsächlich gebotenen Preises höheren Angebotspreis haben.

\section{Die alternative Bemessungsgrundlage: Preise außerhalb des Landes}

Eine andere Möglichkeit ist, sich auf Bedingungen außerhalb Chinas zu verlassen. Sowohl das WTO-Berufungsgremium als auch die EU-Kommission haben in Erwä-

63 Vorschlag für eine Verordnung des Europäischen Parlaments und des Rates, über den Zugang von Waren und Dienstleistungen aus Drittländern zum EU-Binnenmarkt für das öffentliche Beschaffungswesen und über die Verfahren zur Unterstützung von Verhandlungen über den Zugang von Waren und Dienstleistungen aus der Union zu den öffentlichen Beschaffungsmärkten von Drittländern, $\operatorname{COM}(2012) 124$ final.

64 Geänderter Vorschlag für eine Verordnung des Europäischen Parlaments und des Rates, über den Zugang von Waren und Dienstleistungen aus Drittländern zum EU-Binnenmarkt für öffentliche Aufträge und über die Verfahren zur Unterstützung von Verhandlungen über den Zugang von Waren und Dienstleistungen aus der Union zu den Märkten für öffentliche Aufträge von Drittländern, $\operatorname{COM}(2016) 34$ final. 
gung gezogen, dass normale Formen der Anpassungen von Bemessungsgrundlagen nicht praktikabel und daher nicht sinnvoll sind. Die Verwendung von Drittlandspreisen ist daher die bevorzugte Variante.

Innerhalb der Grenzen von Abschnitt 15(b) CAP können diese alternativen Bemessungsgrundlagen auf unterschiedliche Weise angewandt und zudem mit der Bedingung verknüpft werden, dass China bestimmte Bedenken oder Schwierigkeiten, die die Kommission bei der Durchführung erfolgreicher Antisubventionsuntersuchungen festgestellt hat, ausräumt.

Die möglichen Formen der Anwendung alternativer Bemessungsgrundlagen können z.B. wie folgt gestaltet werden:

- Anwendung von Drittlandspreisen: Die derzeitige Praxis nach Artikel 6(d)(ii) Antisubventionsverordnung, auf vergleichbare Drittlandspreise zurückzugreifen, kann unter Anwendung von Abschnitt 15(b) CAP auf jede Art von Subventionen ausgeweitet werden. Das Berufungsgremium hat diesen Mechanismus bereits in seiner Rechtsprechung gebilligt und auf Subventionen in Form der Bereitstellung von Waren oder Dienstleistungen oder des Kaufs von Waren durch eine Regierung ausgedehnt und die Möglichkeit der Verwendung von Bemessungsgrundlagen aus Drittländern auch auf Darlehen ausgeweitet. Die Kommission verwendet in der Regel die auf den Märkten Taiwans oder Hongkongs geltenden Bedingungen als vergleichbare Drittlandspreise. Diese stehen der Kommission als „Out-of-Country-Benchmark" zur Verfügung, wenn sie Antisubventionsuntersuchungen in Bezug auf chinesische Importe durchführt. Während die weitere Ausweitung auf die anderen Subventionsarten nach Artikel 6 Antisubventionsverordnung oder Artikel 14 des Übereinkommens im Falle von sonstigen Exportstaaten von der Auslegung des Berufungsgremiums abhängt, können im Falle von China nach $\mathrm{Ab}$ schnitt 15(b) CAP alle Arten von Subventionen erfasst werden.

- Anwendung der Weltmarktpreise: Die Weltmarktpreise sind nach Artikel 6 Buchstabe d) Ziffer ii) Antisubventionsverordnung auch als Out-of-CountryBenchmarks zulässig, aber bisher hat sich die Kommission nicht dafür entschieden, in Fällen, die subventionierte chinesische Einfuhren betreffen, Weltmarktpreise heranzuziehen. Diese Weltmarktpreise müssen dann alle Länder enthalten, die zur globalen Preisbildung beitragen; Preise, die ihren Ursprung in verzerrten Märkten haben, sollten von einer solchen Preisbildung ausgeschlossen werden. ${ }^{65}$

- Verwendung von Preisfaktoren aus Drittländern: Die Bemessungsgrundlage kann auch durch die Verwendung von Ersatzwerten/Preisen auf einer Faktor-fürFaktor-Basis für jeden Input konstruiert werden, die dann summiert werden („surrogate constructed value"); diese Methode findet sich im US-Antidumpingrecht. ${ }^{66}$ Diese (aufwendige) Methode kann natürlich zu sehr hohen Vergleichspreisen und damit auch zu extrem hohen Ausgleichszöllen führen.

- Preise basierend auf einem Private Investor Test (PIT): Die Berechnung der Höhe der Subvention im Hinblick auf den vom Staat gewährten Vorteil kann auf die Höhe 
der Subvention oder des Betrages festgelegt werden, die ein privater Investor zu gewähren bereit gewesen wäre. Dieser Private Investor-Test bzw. die Prüfung der wirtschaftlichen Angemessenheit basiert auf dem beihilfenrechtlichen Prinzip des Marktinvestors (market investor principle). Die EU hat auf der Grundlage dieses Tests in der Sache $E C-D R A M s$ argumentiert. Das Panel bestätigte die Position der $\mathrm{EU}^{67}$ und dies wurde in der späteren Panel-Entscheidung Japan-DRAMs, die sich mit demselben Argument befasste, nicht aufgehoben. ${ }^{68}$

\section{Weitere nicht vom CAP abgedeckte Maßnahmen zur Effektivierung des Antisubventionsmechanismus}

Während einige praktische Schwierigkeiten für den Abschluss der Antisubventionsuntersuchungsverfahren für China jedenfalls theoretisch gelöst werden können, werden noch weitere Vorschläge gemacht, die wohl nicht unter das CAP und hier speziell Abschnitt 15(b) CAP fallen. Sie beziehen sich erstens auf die Definition der öffentlichen Körperschaft, zweitens auf den Mangel an Transparenz und drittens auf den allgemeinen Verlauf der Untersuchung.

Das WTO-Berufungsgremium hat eine enge Auslegung der „öffentlichen Körperschaft" im Sinne des Übereinkommens über Subventionen und Ausgleichsmaßnahmen vorgenommen. Diese vielfach kritisierte Interpretation erschwert bekanntlich die Anwendung von Ausgleichszöllen gegen Maßnahmen, die von staatlichen Behörden ausgehen. ${ }^{69}$ Auch wenn China inzwischen vielen staatlichen Unternehmen wichtige staatliche Funktionen zuweist und in ihnen Parteivertreter einsetzt - was es einfacher macht zu argumentieren, dass diese Unternehmen als öffentliche Körperschaften anzusehen sind, die staatliche Autorität und Kontrolle ausüben -, sollte es eine Möglichkeit geben zu definieren, dass staatliche Unternehmen ebenso wie Staatsfonds (Sovereign Wealth Funds, SWF) im Falle Chinas öffentliche Körperschaften sind, es sei denn, diese Unternehmen weisen nach, dass sie keine öffentliche Gewalt ausüben (Beweislastumkehr). Diese Konzeption fand in der Literatur Unterstützung, ${ }^{70}$ wurde aber vom Berufungsgremium nicht geteilt. ${ }^{71} \mathrm{Ob}$ dieser Ansatz dennoch unter der Anwendung des CAP verfolgt werden kann, ist fraglich und es bleibt daher abzuwarten, ob er von der WTO-Streitbeilegung akzeptiert wird. Ganz allgemein würde die Erweiterung der Definition von „Regierung oder öffentliche Stelle“ die Anwen-

67 Panel report, European Communities - Countervailing Measures on Dynamic Random Access Memory Chips from Korea, WT/DS299/R, 03.08.2005, para. 7.205.

68 Panel report, Japan - Countervailing Measures on Dynamic Random Access Memory Chips from Korea, WT/DS336/R, 17.12.2007, para. 7.275.

69 S. Wu, HILJ 2016/2, S. 301 ff; Ding, JWT 2014/1, S.188; Bhala/Kim, AJWH 2019/1, S. 19 f. Andere sehen ausreichende Klarheit und Kapazität für die Erfassung von Staatsunternehmen: Zhou/Goa/Bai, ICLQ 2019/4, S. 1017 ff; Chiang, GJIL 2017/2, S. 873.

70 Cartland/Deparye/Woznowski, JWT 2012/5, S. 1011.

71 Appellate Body report, United States - Definitive Anti-Dumping and Countervailing Duties on Certain Products from China, WT/DS379/AB/R, 25.03.2011, para. 352. 
dung von Handelsschutzmaßnahmen erleichtern, zumindest in den Fällen, in denen das Produkt direkt aus China importiert wird. ${ }^{72}$

Die EU hat vorgeschlagen, eine widerlegbare Vermutung aufzunebmen, dass alle nicht notifizierten Subventionen als anfechtbar gelten, dies zumindest nach einem erfolglosen Verfahren gemäß Artikel 25.10 des Übereinkommens über Subventionen und Ausgleichsmaßnahmen. ${ }^{73}$ Außerdem hat sie ein abgestuftes System mit "Strafen“ nach Jahren der Nichtnotifizierung vorgeschlagen, die von der Pflicht zur Beantwortung von Fragen nach zwei Jahren bis zur Qualifikation als „inaktives Mitglied“ im SCM-Ausschuss mit eingeschränktem Rederecht nach drei Jahren reichen. ${ }^{74}$ Das WTO-Sekretariat könnte eine solche negative Notifizierungsbilanz in die Trade Policy Review Mechanism (TPRM)-Berichte aufnehmen. ${ }^{75}$

Fraglich ist, ob diese Vorschläge als ausreichend für eine effektivere Untersuchung staatlicher Subventionen angesehen werden können. Was auch notwendig sein könnte, um die Transparenz der chinesischen Subventionsprogramme zu verbessern, ist die Festlegung verbindlicher Fristen und Sanktionen für den Fall, dass diese Subventionsprogramme nicht beim SCM-Ausschuss angezeigt werden.

Darüber hinaus können die folgenden Überlegungen berücksichtigt werden, wenn es um die Zukunft von Antisubventionsuntersuchungen gegen chinesische Importe geht:

- Erfolgreiche Rechtsmittel rückwirkend anwenden: Eine weitere Option könnte darin bestehen, von den Empfängern eines Zuschusses die Rückzahlung des gesamten Zuschussbetrags zu verlangen. ${ }^{76}$ Dies ist gängige Praxis in der EU, aber es ist fraglich, ob eine solche Praxis auch von Abschnitt 15(b) CAP abgedeckt wird.

- Untersuchungen von Amts wegen oder anonyme Beschwerden: Viele Beschwerdeführer befürchten bei der Anfechtung staatlicher Subventionen zusätzliche Vergeltungsmaßnahmen außerhalb des WTO-Systems durch China. ${ }^{77}$ Wenn man von der Kommission eingeleitete Untersuchungen von Amts wegen oder anonyme Beschwerdeverfahren zuließe, könnten die Befürchtungen von Industrieorganisationen, die Untersuchungen einleiten wollen, entgegnet werden.

- Kapitel zu staatlichen Unternehmen und ein neues plurilaterales Antisubventions-/Ausgleichszoll-Abkommen: Einige argumentieren, dass ein neues Antisubventions-/Ausgleichszoll-Abkommen mit einem erweiterten und eindeutigen Anwendungsbereich einschließlich spezifischer Regeln für staatliche Unternehmen erforderlich sei. ${ }^{78}$ Bown plädiert für durchsetzbare Regeln, die alle wirtschaftlichen

72 Ding, JWT 2014/1, S. 183.

73 EU concept paper on WTO reform, Juli 2018. See also Hu, CEPS Policy Insights No. 2019/16, S. $19 \mathrm{f}$.

74 EU concept paper on WTO reform, Juli 2018.

75 Kazeki, GTCJ 2010/5, S. $191 \mathrm{ff}$.

76 Panel report, Australia - Subsidies Provided to Producers and Exporters of Automotive Leather, WT/DS126/RW, 21.01.2000, paras 6.39ff.

77 Hierzu auch T.Müller-Ibold, in diesem Heft, S. 239 ff.

78 Bown, Anhörung vor der U.S.-China Economic and Security Review Commission, 08.06.2018. 
Verzerrungen aufgrund staatlicher Unternehmen angehen: Einerseits sollte es durchsetzbare Regelungen für staatliche Unternehmen vorsehen, andererseits und im Gegenzug würde es China erlaubt sein, seine staatlichen Unternehmen zu behalten. ${ }^{79}$ Darüber hinaus können Kapitel über staatliche Unternehmen Mängel des Übereinkommens über Subventionen und Ausgleichsmaßnahmen und auf die Kritik an der Rechtsprechung des Berufungsgremiums eingehen. ${ }^{80}$ So enthält beispielsweise das neue Comprebensive and Progressive Agreement for Trans-Pacific Partnership (CPTPP) ein Kapitel über staatliche Unternehmen und bestimmte Monopole (Kapitel 17), und auch die EU führt in ihre Freihandelsabkommen Kapitel über staatliche Unternehmen ein.

\section{F. Schlussfolgerungen und Ausblick}

Der Verzicht auf die Drittlandsmethode in Antidumpinguntersuchungen schließt nicht aus, dass ähnliche Methoden für Gegenmaßnahmen im Antisubventionsbereich nunmehr entwickelt werden. Umfassendere Ausgleichsmaßnahmen könnten eine Option sein - wurden aber bislang in den Diskussionen über die Zuerkennung des Marktwirtschaftsstatus an China oder die Berechnung von Antidumpingzöllen nicht umfassend berücksichtigt. Abschnitt 15(b) des WTO-Beitrittsprotokolls Chinas könnte daher in Verbindung mit den bestehenden WTO-Regeln für Subventionen eine zukünftige Möglichkeit des Schutzes vor Chinas „einmaligem Wirtschaftsmodell“ bieten. Derzeit hat die EU jedoch noch immer nur wenige Antisubventionsuntersuchungen gegen China eingeleitet.

Die WTO-Mitglieder sollten damit beginnen, gut koordinierte Ausgleichsuntersuchungen durchzuführen, und parallel dazu Verfahren bei der WTO einleiten, ${ }^{81}$ um Chinas Subventionen und staatliche Marktinterventionen durch Staatsunternehmen zu begegnen, um deutlich zu machen, wo das WTO-System die Grenzen der Anwendung von Abschnitt 15(b) CAP setzt. Diese Bestimmung hat in ihrer Anwendung mehrere Vorteile im Vergleich zu den normalen Berechnungsregeln des Artikels 14 des Übereinkommens über Subventionen und Ausgleichsmaßnahmen und den Antidumpingregeln von Abschnitt 15(a) CAP: Es kann keine Diskussion über das Auslaufen der Regelung geführt werden, da es keine 15-Jahres-Frist gibt; die Schwelle, die man zur Anwendung der Regelung zu überschreiten hat, ist niedrig - es müssen nur „besondere Schwierigkeiten“ bestehen, und die Methode für die Berechnung der Begünstigung ist völlig offen. Vor diesem Hintergrund muss man sich allerdings fragen, warum diese alternative Möglichkeit des Handelsschutzes bislang noch nicht intensiver diskutiert wurde.

In diesem Papier wird vorgeschlagen, Abschnitt 15(b) CAP wiederholt zu nutzen, um zwei praktische Hürden zu überwinden, die für die Durchführung von Antisub-

79 Ibid.

80 S. Lee, AJWH 2019/1, S. 33-72; Willemyns, JIEL 2016/3, S. 657-680; Matsushita, in: Chaisse et al. (Hrsg.), S. 187-203.

81 Hillman, Anhörung vor der U.S.-China Economic and Security Review Commission, 08.06.2018. 
ventionsuntersuchungen herausgestellt wurden, nämlich die problematische Definition der öffentlichen Körperschaft und die allgemeine Intransparenz der chinesischen Wirtschaft. Darüber hinaus werden auch Alternativen für die Anpassung und Berechnung von Berechnungsgrundlagen vorgeschlagen:

- Immer wenn ein staatliches Unternehmen nicht nachweisen kann, dass es keine staatliche Kontrolle oder Einflussnahme gibt, steht das Kriterium der „besonderen Schwierigkeit" als Einstieg in eine alternative Berechnungsgrundlage offen. Dies bedeutet, dass Abschnitt 15(b) CAP eine diametrale Änderung der Beweislast zulässt, was in dann ermöglicht, eine widerlegbare Vermutung einzuführen. Wenn sich die Möglichkeit für die Heranziehung von Abschnitt 15(b) CAP eröffnet, weil China oder chinesische Unternehmen nicht nachweisen können, dass es keine besonderen Schwierigkeiten gibt, ist eine Anpassung der Berechnungsgrundlage zulässig.

- Eine Anpassung könnte einen sektorspezifischen festen „Aufschlag“ auf den Exportpreis einer Ware vorsehen - es sei denn, der Importeur kann nachweisen, dass eine niedrigere Berechnungsgrundlage für die Berechnung von Ausgleichszöllen gelten sollte.

- Wenn eine Anpassung nicht möglich ist, könnten Berechnungsgrundlagen aus Drittländern oder die Weltpreise für die Berechnung der Vorteile gelten, oder es könnte innerhalb einer alternativen Methode zur Berechnung von Begünstigungen ein faktorbezogener Ansatz verwendet werden.

Dies sind einige Ansatzpunkte, die in der laufenden Auseinandersetzung über die Steigerung der Wirksamkeit von Ausgleichszöllen - auch im Rahmen der Europäischen Industriestrategie - diskutiert werden können.

\section{BIBLIOGRAPHIE}

AEGIS EUROPE, A Pragmatic Approach to China MES: Wait for the WTO to Decide. Why "mitigation options" don't work, the risks of a unilateral interpretation of the Protocol and the key pillars of an effective anti-dumping system, abrufbar unter: https://static1.squarespace.com/static/5537b2fbe4b0e49a1e30c01c/t/56950f fca12f44b2ebOfc7b2/1452609533236/A+PRAGMATIC+APPROACH+TO+CH INA+MES.pdf (31.01.2020)

BHALA RAJ; KIM, NATHAN DEUCKJOO DJ, The WTO's Under-Capacity to Deal with Global Over-Capacity, Asian Journal of WTO \& International Health Law and Policy, 2019, Vol. 14(1), S. 1-32

BOWN, CHAD P., Anhörung vor der U.S.-China Economic and Security Review Commission, 08.06.2018

BOWN, CHAD P.; HILLMAN, JENNIFER A., WTO'ing a Resolution to the China Subsidy Problem, Journal of International Economic Law, 2019, Vol. 22(4), S. 557-578 
BUNDESVERBAND DER DEUTSCHEN INDUSTRIE, Partner and Systemic Competitor - How Do We Deal with China's State-Controlled Economy?, BDI Policy Papers China, Januar 2019

CARTLAND, MICHEL; DEPARYE, GÉRARD; WOZNOWSKI, JAN, Is Something Going Wrong in the WTO Dispute Settlement?, Journal of World Trade, 2012, Vol. 46(5), S. 979-1016

CHIANG, TING-WEI, Chinese State-owned Enterprises and WTO's Anti-subsidy Regime, Georgetown Journal of International Law, 2017, Vol. 49(2), S. 845-886

CHU, C.Y. CYRUS; LEE, PO-CHING, Three Changes Not Foreseen by WTO Rules Framers Twenty-Five Years Ago, Journal of World Trade, 2019, Vol. 53(6), S. 895-922

DING, RU, Public Body' or Not: Chinese State-Owned Enterprise, Journal of World Trade, 2014, Vol. 48(1), S. 167-190

HILLMAN, JENNIFER A., Anbörung vor der U.S.-China Economic and Security Review Commission, 08.06.2018

HU, WEINIAN, China as a WTO developing member, is it a problem?, CEPS Policy Insights No. 2019/16, November 2019

KAZEKI, JUN, The "Middle Pillar": Transparency and Surveillance of Subsidies in the SCM Committee - Reflections after the global Economic Crisis, Global Trade and Customs Journal, 2010, Vol. 5(5), S. 191-198

KELLY, BRIAN D., The Offsetting Duty Norm and the Simultaneous Application of Countervailing and Antidumping Duties, Global Economy Journal, 2011, Vol. 11(2), S. 1-31

LEE, JAEMIN, Trade Agreements' New Frontier - Regulation of State-Owned Enterprises and Outstanding Systemic Challenges, Asian Journal of WTO \& International Health Law and Policy, 2019, Vol. 14(1), S. 33-72

MATSUSHITA, MITSUO, State-Owned Enterprises in the TPP Agreement, in: Chaisse, Julien et al. (Hrsg.), Paradigm Shift in International Economic Rule-making: TPP as a New Model for Trade Agreements?, Springer, 2017, S. 187-203

MÜLLER, SOPHIA, The Prospects of EU Trade Defence against Chinese Imports after 2016 - Taming the Dragon through Countervailing Duties?, 2015, abrufbar unter: http://ssrn.com/abstract=2665960 (31.01.2020)

MÜLLER, SOPHIA, The Use of Alternative Benchmarks in Anti-Subsidy Law: A Study on the WTO, the EU and China, Springer, 2018

MÜLLER, SOPHIA, Anti-Subsidy Investigations Against China: The "Great Leap Forward" in Reforming EU Trade Defence?, in: Bungenberg, Marc et al. (Hrsg.), European Yearbook of International Economic Law 2017, Springer, 2018, S. $125-155$ 
MÜLLER, WOLFGANG, The EU's Trade Defence Instruments: Recent Judicial and Policy Developments, in: Bungenberg, Marc et al. (Hrsg.), European Yearbook of International Economic Law 2017, Springer, 2018, S. 205-225

QIN, JULIA YA, WTO Regulation of Subsidies to State-Owned Enterprises (SOEs) - A Critical Appraisal of the China Accession Protocol, Journal of International Economic Law, 2004, Vol. 7(4), S. 863-919

UNITED STATES TRADE REPRESENTATIVE, 2018 Report to Congress On China's WTO Compliance, Februar 2019, abrufbar unter: https://ustr.gov/sites/d efault/files/2018-USTR-Report-to-Congress-on-China\%27s-WTO-Compliance. pdf (31.01.2020)

VAN BAEL \& BELLIS, EU Anti-Dumping and Other Trade Defence Instruments, Kluwer, 2019

VERMULST, EDWIN; GATTA, BRIAN, Concurrent Trade Defence Investigations in the EU, the EU's new anti-subsidy practice against China, and the Future of Both, World Trade Review, 2012, Vol. 11(3), S. 527-553

WILLEMYNS, INES, Disciplines on State-Owned Enterprises in International Economic Law: Are We Moving in the Right Direction?, Journal of International Economic Law, 2016, Vol. 19(3), S. 657-680

WU, MARK, The "China, Inc." Challenge to Global Trade Governance, Harvard International Law Journal, 2016, Vol. 57(2), S. 261-342

ZHOU, WEIHUAN/GOA, HENRY; BAI, XUE, Building A Market Economy Through WTO-Inspired Reform of State-Owned Enterprises in China, International and Comparative Law Quarterly, 2019, Vol. 68(4), S. 977-1022

\section{Annexe}

Annex 1: Überblick über AS-Untersuchungen gegen China

\begin{tabular}{|l|l|l|l|l|}
\hline Nr. & $\begin{array}{l}\text { Untersuchtes } \\
\text { Produkt }\end{array}$ & Land & $\begin{array}{l}\text { Endgültige } \\
\text { AS-Verordnung }\end{array}$ & $\begin{array}{l}\text { Veröffent- } \\
\text { lichung }\end{array}$ \\
\hline AS557 & $\begin{array}{l}\text { Gestrichenes Fein- } \\
\text { papier }\end{array}$ & China & $\begin{array}{l}\text { Durchführungs-ver- } \\
\text { ordnung (EU) } \\
\text { Nr. 452/2011 des Ra- } \\
\text { tes vom 6. Mai 2011 }\end{array}$ & $\begin{array}{l}\text { ABl. L 128, } \\
\text { S. 18ff } \\
(14.5 .2011)\end{array}$ \\
\hline AS564 & WWAN-Modems & China & [beendet] & $\begin{array}{l}\text { AB1. L 58, } \\
\text { S. 36ff } \\
(3.3 .2011)\end{array}$ \\
\hline AS587 & $\begin{array}{l}\text { Organisch be- } \\
\text { schichteter Stahler- } \\
\text { zeugnisse }\end{array}$ & China & $\begin{array}{l}\text { Durchführungs-ver- } \\
\text { ordnung (EU) } \\
\text { Nr. 215/2013 des Ra- } \\
\text { tes vom. 11. März } \\
\text { 2013 }\end{array}$ & $\begin{array}{l}\text { ABl. L 73, 16ff } \\
\text { (15.3.2013) }\end{array}$ \\
\hline
\end{tabular}




\begin{tabular}{|c|c|c|c|c|}
\hline $\mathrm{Nr}$. & $\begin{array}{l}\text { Untersuchtes } \\
\text { Produkt }\end{array}$ & Land & \begin{tabular}{|l} 
Endgültige \\
AS-Verordnung
\end{tabular} & $\begin{array}{l}\text { Veröffent- } \\
\text { lichung }\end{array}$ \\
\hline AS589 & Fahrräder & China & [beendet] & $\begin{array}{l}\text { ABl. L 136, } \\
\text { S. } 15 \mathrm{ff} \\
(23.5 .2013)\end{array}$ \\
\hline AS594 & Solarplatten & China & $\begin{array}{l}\text { Durchführungs-ver- } \\
\text { ordnung (EU) } \\
\text { Nr.1239/2013 des } \\
\text { Rates vom 2. De- } \\
\text { zember } 2013\end{array}$ & $\begin{array}{l}\text { ABl. L 325, } \\
\text { S. } 66 \mathrm{ff} \\
(5.12 .2013)\end{array}$ \\
\hline AS599 & Solarglas & China & $\begin{array}{l}\text { Durchführungs-ver- } \\
\text { ordnung (EU) } \\
\text { Nr. 471/2014 der } \\
\text { Kommission vom } \\
\text { 13. Mai } 2014\end{array}$ & $\begin{array}{l}\text { ABl. L 142, } \\
\text { S. } 23 \mathrm{ff} \\
(14.5 .2014)\end{array}$ \\
\hline AS603 & $\begin{array}{l}\text { Waren aus Glasfa- } \\
\text { serfilamenten }\end{array}$ & China & $\begin{array}{l}\text { Durchführungs-ver- } \\
\text { ordnung (EU) } \\
\text { Nr.1379/2014 der } \\
\text { Kommission vom } \\
\text { 16. Dezember } 2014\end{array}$ & $\begin{array}{l}\text { ABl. L 367, } \\
\text { S. } 22 \mathrm{ff} \\
(23.12 .2014)\end{array}$ \\
\hline AS634 & $\begin{array}{l}\text { Warmgewalzter } \\
\text { Flacherzeugnisse } \\
\text { aus Eisen und Stahl }\end{array}$ & China & $\begin{array}{l}\text { Durchführungs-ver- } \\
\text { ordnung (EU) } \\
\text { 2017/969 der Kom- } \\
\text { mission vom 8. Juni } \\
2017 \\
\end{array}$ & $\begin{array}{l}\text { ABl. L 146, } \\
\text { S. } 17 \mathrm{ff} \\
(9.6 .2017)\end{array}$ \\
\hline AS641 & $\begin{array}{l}\text { Luftreifen aus Kau- } \\
\text { tschuk für Omni- } \\
\text { busse und Kraft- } \\
\text { fahrzeuge }\end{array}$ & China & $\begin{array}{l}\text { Durchführungs-ver- } \\
\text { ordnung (EU) } \\
\text { 2018/1690 der Kom- } \\
\text { mission vom 9. No- } \\
\text { vember } 2018\end{array}$ & $\begin{array}{l}\text { ABl. L 283, } \\
\text { S. } 1 \mathrm{ff} \\
(12.11 .2018)\end{array}$ \\
\hline AS646 & Elektrofahrräder & China & $\begin{array}{l}\text { Durchführungs-ver- } \\
\text { ordnung (EU) } \\
\text { 2019/72 der Kom- } \\
\text { mission vom 17. Ja- } \\
\text { nuar } 2019\end{array}$ & $\begin{array}{l}\text { ABl. L 16, } \\
\text { S. } 5 \mathrm{ff} \\
(18.1 .2019)\end{array}$ \\
\hline AS656 & Glasfasermatten & $\begin{array}{l}\text { China und } \\
\text { Ägypten }\end{array}$ & [unerledigt] & $\begin{array}{l}\text { ABl. C 167, } \\
\text { p. } 11 \mathrm{ff} \\
(16.5 .2019)\end{array}$ \\
\hline AS660 & $\begin{array}{l}\text { Warmgewalzter } \\
\text { Flacherzeugnisse } \\
\text { aus nicht rostendem } \\
\text { Stahl }\end{array}$ & $\begin{array}{l}\text { China und } \\
\text { Indonesien }\end{array}$ & [unerledigt] & $\begin{array}{l}\text { ABl. C 342, } \\
\text { p. } 18 \mathrm{ff} \\
(10.10 .2019)\end{array}$ \\
\hline
\end{tabular}


Annex 2: Artikel 10 Antisubventionsverordnung Untersuchung ${ }^{82}$

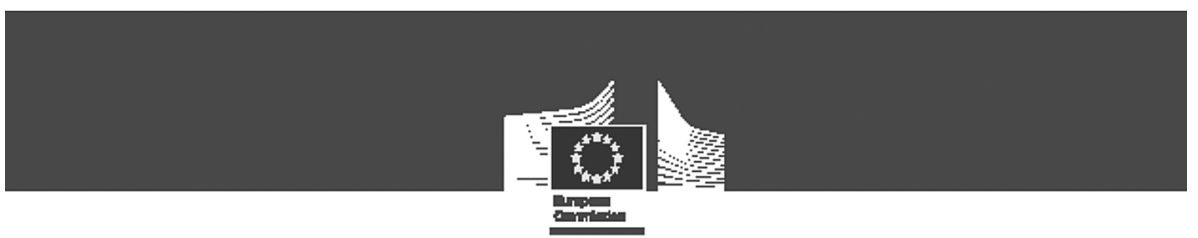

Anti-subsidy Article 10 Investigation

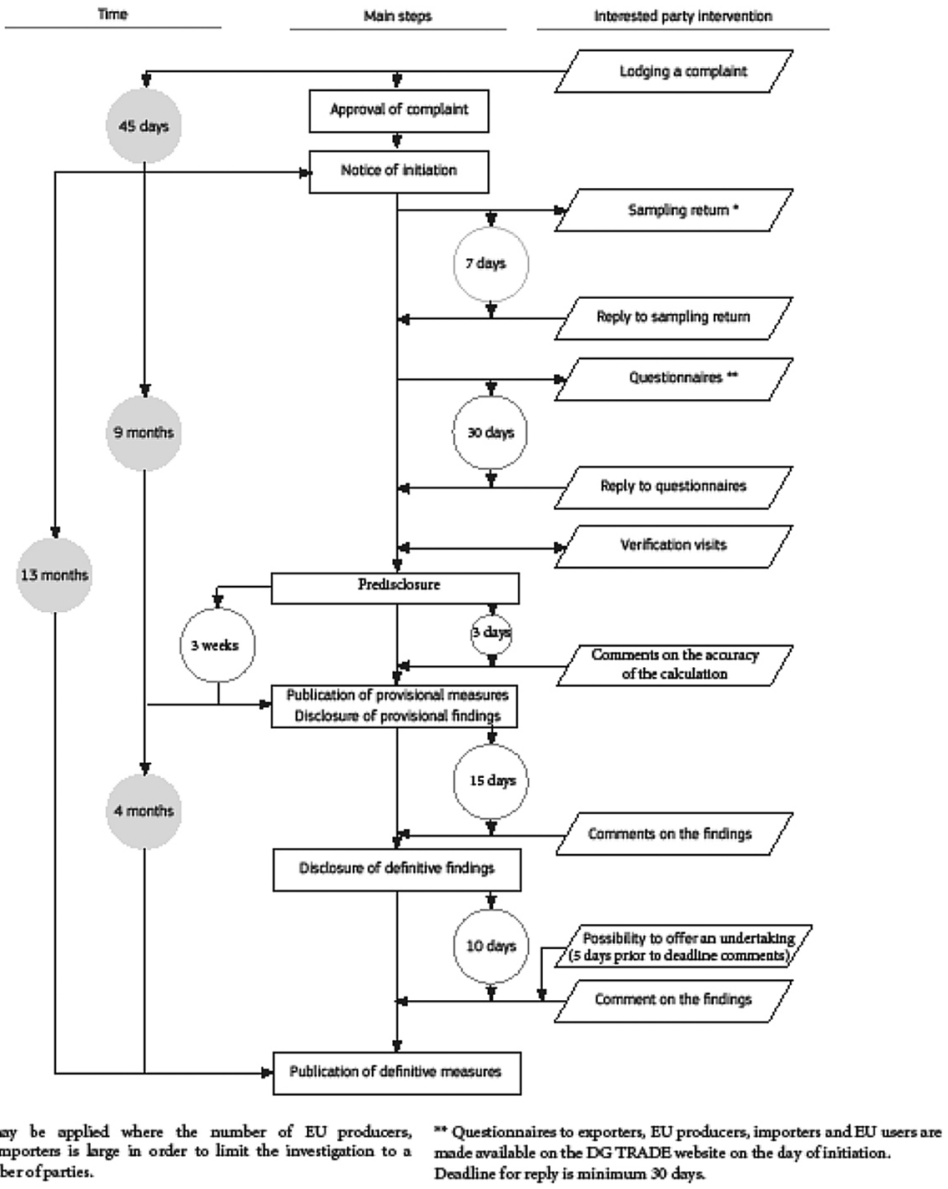

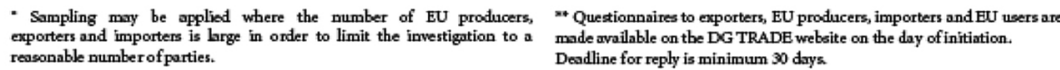

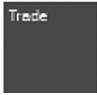

82 Quelle: Europäische Kommission, Flowchart of the anti-subsidy investigation process, 12.09.2019, abrufbar unter: https://trade.ec.europa.eu/doclib/docs/2013/april/tradoc_151 025.pdf (11.03.2020). 
